\title{
Modelamento não Linear de Dois Elos de um Robô Eletromecânico de Cinco Graus de Liberdade
}

\author{
José Antônio Riul ${ }^{1}$ \\ Departamento de Engenharia Mecânica, Centro de Tecnologia, UFPB, João Pessoa, PB \\ Paulo Henrique de Miranda Montenegro ${ }^{2}$ \\ Departamento de Engenharia Mecânica, Centro de Tecnologia, UFPB, João Pessoa, PB
}

\begin{abstract}
Resumo. O objetivo do presente trabalho é o modelamento não linear de dois elos de um robô manipulador eletromecânico de cinco graus de liberdade (5 GDL). O robô manipulador é composto por cinco juntas rotacionais, por quatro elos e por uma garra. Cinco motores de corrente contínua são utilizados para o acionamento do robô e a transmissão do movimento dos motores para as juntas é realizada através de trens de engrenagens. As medidas das posições angulares das juntas são realizadas por potenciômetros. Modelos de robôs manipuladores são obtidos usando equações de Newton - Euler ou de Lagrange; e são acoplados e não lineares. Neste trabalho, um modelo não linear é obtido, usando a série de Volterra em tempo discreto; e os parâmetros do robô manipulador são identificados, através do algoritmo Least Mean Squares (LMS), com base nas excitações e respostas dos elos do robô. Finalizando, são apresentados resultados obtidos através do modelo gerado.
\end{abstract}

Palavras-chave. Sistema não linear, Robótica, Identificação

\section{Introdução}

O presente trabalho tem como objetivo o modelamento não linear de dois elos de um robô manipulador eletromecânico de cinco graus de liberdade (5 GDL). Os elos 1 e 2 a serem controlados são mostrados na Fig. 1. O modelo matemático de um sistema pode ser obtido através de leis físicas, conhecido como modelo caixa branca ou por técnica de identificação paramétrica, conhecida como modelo caixa preta, que depende de dados reais do sistema. Modelos caixa branca de robôs manipuladores são não lineares [5, 10], enquanto que a identificação caixa preta, gera modelos lineares e não lineares [1], que podem ser usados para projeto e implementação de controladores adaptativos. Os modelos caixa branca, exigem elevado esforço computacional $[8,11]$. Na utilização de modelos caixa preta, suas estruturas são definidas a priori, e com isto, a escolha de modelos de primeira ou segunda ordem, que representam bem os sistemas reais, e que requerem baixo esforço computacional,

\footnotetext{
${ }^{1}$ riul@ct.ufpb.br

2 paulo@ct.ufpb.br
} 
são empregados. Modelos caixa preta, não lineares, considerando o acoplamento entre os elos 1 e 2 do robô serão obtidos usando o algoritmo Least Mean Squares (LMS). Finalizando, são apresentados resultados obtidos através do modelo gerado.

\section{Descrição do Sistema}

O Robô Manipulador, mostrado na Fig. 1 é um robô didático, pesando aproximadamente $7 \mathrm{~kg}$, referência RD5NT, fabricado pela empresa Didacta Itália, é composto de cinco juntas rotativas, quatro elos e uma garra. A primeira junta rotativa refere-se ao movimento angular da base, com deslocamento máximo de $293^{\circ}$, a segunda junta rotativa refere-se ao ombro, com deslocamento angular máximo de $107^{\circ}$, a terceira junta rotativa refere-se ao cotovelo, com deslocamento angular máximo de $284^{\circ}$, a quarta junta rotativa refere-se ao pulso com deslocamento angular máximo de $360^{\circ}$ e a quinta junta rotativa refere-se a um sistema corôa / parafuso sem fim, responsável pelo curso da garra, de no máximo $22 \mathrm{~mm}$, capacidade de fixação de carga de 350 gramas e parada automática por meio de uma micro chave operando com velocidade de fechamento ajustável.

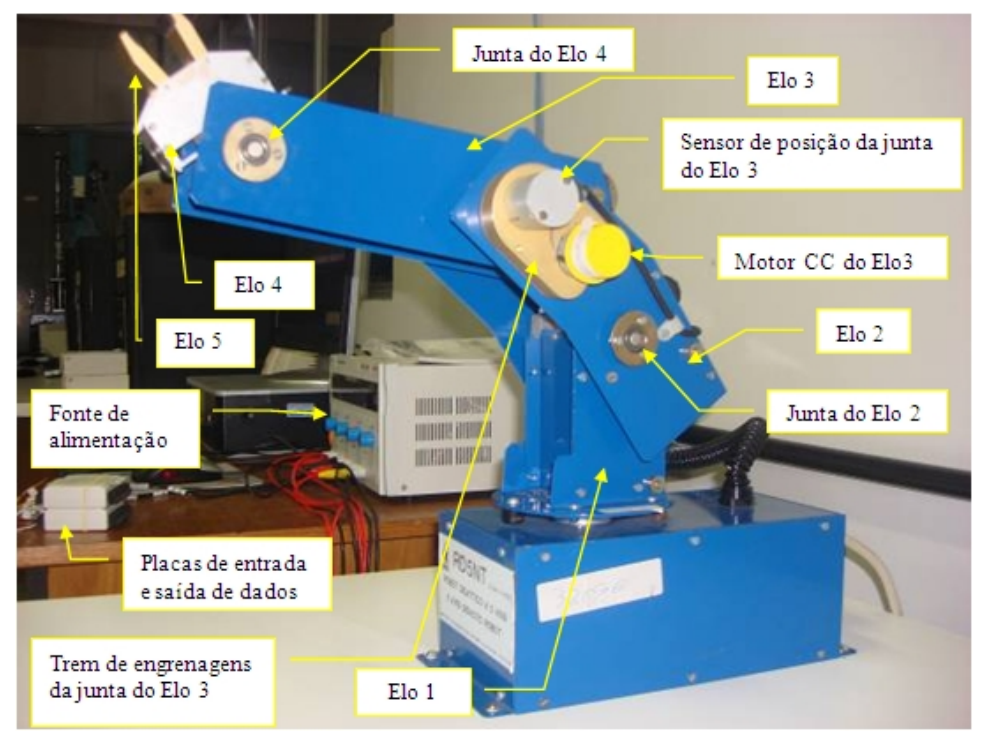

Figura 1: Robô manipulador de cinco gdl.

Os elos do robô manipulador representam o tronco, o braço, o antebraço e o punho. A transmissão de cada movimento é feita por meio de bloco motor redutor, com dois estágios de redução, e com relação de transmissão total de 1/500. Os motores dos blocos são de corrente continua, referência 2139.906-22.112-050, fabricados pela Maxon Motor, com potencia de 2,5 watts e com capacitor de longa vida. A voltagem nominal dos motores CC é de 12 volts e a rotação máxima sem carga é de $6480 \mathrm{rpm}$. A reprodução dos deslocamentos angulares das juntas e movimento da garra é assegurada por meio de potenciômetros rotativos lineares, referência 78CSB502, fabricados pela Sfernice, com resistência de $5 \mathrm{k} \Omega$. Um computador HP Compaq com processador AMD Athlon dual core de $985 \mathrm{Mhz}$ e 786 
MB de RAM é utilizado para enviar comando de acionamento aos motores $\mathrm{CC}$ e receber os sinais dos sensores potenciométricos.

A comunicação do robô com o computador, é realizada através de duas placas de entrada e saída de dados, NI USB-6009 e de um programa computacional nas plataformas LabView e Matlab.

\section{Identificação Não Linear do Robô Manipulador}

A identificação de sistemas é uma área do conhecimento que estuda técnicas alternativas de modelagem matemática $[3,4,6,9]$. Uma das características dessas técnicas é que pouco ou nenhum conhecimento prévio do sistema é necessário e, consequentemente, tais métodos são referidos como modelagem (ou identificação) caixa preta ou modelagem empírica [2].

Modelos matemáticos não lineares de sistemas, podem ser obtidos utilizando-se a série de Volterra [7]. O modelo paramétrico de Volterra mostrado na Eq. (1), é apropriado para estimação de parâmetros tendo como base os sinais de entrada e de saída de um sistema de uma entrada e uma saída (SISO).

$$
\begin{aligned}
& v(k)+\sum_{i=1}^{m} a_{N i} v(k-i)+\sum_{\beta=0}^{h} \sum_{i=1}^{m} a_{2 \beta i} v(k-i) v(k-i-\beta)+\ldots \\
& \sum_{\beta_{1}=0}^{h} \sum_{\beta_{2}=\beta_{1}}^{h} \ldots \sum_{\beta_{p-1}=\beta_{p-2}}^{h} \sum_{i=1}^{m} a_{p \beta_{1} \ldots \beta_{p-1}} v(k-i) \prod_{\xi=1}^{p-1} v\left(k-i-\beta_{\xi}\right)=\sum_{i=1}^{m} b_{i} u(k-d-i)+c_{s s}
\end{aligned}
$$

onde: $\mathrm{m}$ - ordem do modelo do sistema;

$\mathrm{d}$ - atraso de transporte;

$\mathrm{p}$ - grau de não linearidade do modelo do sistema;

$\mathrm{h}$ - horizonte;

$\mathrm{c}_{\mathrm{ss}}-$ nível DC;

$\mathrm{a}_{\mathrm{Ni}}$ e $\mathrm{b}_{\mathrm{i}}$ - parâmetros do modelo do sistema;

$\mathrm{k}$ - tempo discreto;

$\mathrm{u}(\mathrm{k})$ - excitação no sistema;

$v(k)$ - resposta do sistema;

Considerando $\mathrm{m}=1, \mathrm{~d}=1, \mathrm{p}=3, \mathrm{~h}=1$ e $c_{s s}=0$ na Eq. (1), obtém-se a Eq. (2), de um modelo não linear, de não linearidade na saída.

$$
\begin{aligned}
& v(k)=\left[\begin{array}{llllllll}
a_{1} & b_{11} & \mathrm{a}_{201} & \mathrm{a}_{211} & \mathrm{a}_{3001} & \mathrm{a}_{3111} & \mathrm{a}_{3101}
\end{array}\right]\left[\begin{array}{llll}
-v(k-1) & u(k-2) & -v^{2}(k-1)
\end{array}\right. \\
& \left.-v(k-1) v(k-2)-v^{3}(k-1)-v(k-1) v^{2}(k-2)-v^{2}(k-1) v(k-2)\right]^{t}
\end{aligned}
$$

Para análise nos dois elos do robô manipulador, e considerando o acoplamento dinâmico entre eles, a Eq. (2) é reescrita conforme a Eq. (3), por se tratar de um sistema de múltiplas 
entradas e de múltiplas saídas (MIMO).

$$
[v(k)]=[\theta(k)][\varphi(k)]
$$

onde: $[v(k)]$ - vetor de saida;

$[\theta(k)]$ - matriz de parâmetros;

$[\varphi(k)]$ - vetor de medidas.

Na Equação (3), a matriz de parâmetros é dada pela Eq. (4), o vetor de medidas dado pela Eq. (5) e o vetor de saída dado pela Eq. (6). O mesmo vetor de medidas é utilizado para obtenção das saídas $v_{1}(k)$ e de $v_{2}(k)$. Na Equação (4) o tempo discreto k dos parâmetros $a_{\mathrm{Ni}}$ e $b_{\mathrm{i}}$ foi omitido.

$$
\begin{aligned}
& {\left[\begin{array}{l}
\theta_{1}(k) \\
\theta_{2}(k)
\end{array}\right]=\left[\begin{array}{llllllllllllll}
a_{1} & b_{1} & a_{2} & a_{3} & a_{4} & a_{5} & a_{6} & a_{7} & b_{2} & a_{8} & a_{9} & a_{10} & a_{11} & a_{12} \\
a_{13} & b_{3} & a_{14} & a_{15} & a_{16} & a_{17} & a_{18} & a_{19} & b_{4} & a_{20} & a_{21} & a_{22} & a_{23} & a_{24}
\end{array}\right]}
\end{aligned}
$$

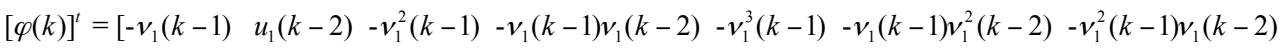

$$
\begin{aligned}
& \left.-v_{2}(k-1) u_{2}(k-2)-v_{2}^{2}(k-1)-v_{2}(k-1) v_{2}(k-2)-v_{2}^{3}(k-1)-v_{2}(k-1) v_{2}^{2}(k-2)-v_{2}^{2}(k-1) v_{2}(k-2)\right] \\
& {[v(k)]^{t}=\left[\begin{array}{ll}
v_{1}(k) & v_{2}(k)
\end{array}\right]}
\end{aligned}
$$

onde: $\theta_{i}(k)$ - parâmetros dos elos 1 e 2 do robô;

$\varphi_{i}(k)$ - vetor de medidas dos elos 1 e 2 do robô,

$v_{i}(k)$ - saídas dos elos 1 e 2 do robô;

$u_{i}(k)$ - entradas nos elos 1 e 2 do robo;

$\mathrm{i}=1$ e 2 .

A identificação tipo caixa preta é utilizada no modelamento não linear dos elos do robô manipulador sob análise, através do algoritmo Least Mean Squares (LMS), dado pela Eq. (7) $[2,4]$.

$$
\hat{\theta}(\mathrm{k}+1)=\hat{\theta}(\mathrm{k})+\gamma(\mathrm{k}+1) \varphi(\mathrm{k}+1) \varepsilon(\mathrm{k}+1)
$$

onde:

$$
\gamma(\mathrm{k}+1)=1 / 1+\varphi^{\mathrm{T}}(\mathrm{k}+1) \varphi(\mathrm{k}+1)
$$




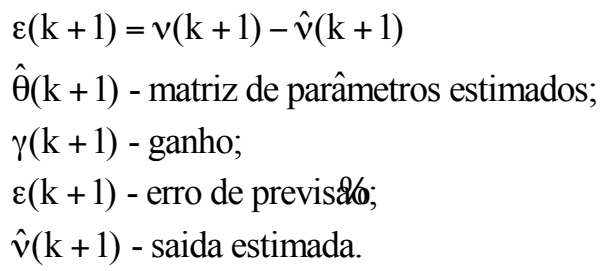

A qualidade do modelo estimado pode ser verificada utilizando várias técnicas, dentre elas para se investigar a magnitude do índice de desempenho tem-se o somatório do erro quadrático (SEQ), dado pela Eq. (10) e o coeficiente de correlação múltipla $\left(\mathrm{R}^{2}\right)$, dado pela Eq. (11) [4].

$$
\begin{aligned}
& \mathrm{SEQ}_{\mathrm{i}}=\sum_{\mathrm{k}=1}^{\mathrm{N}}\left[v_{\mathrm{i}}(\mathrm{k})-\hat{v}_{\mathrm{i}}(\mathrm{k})\right]^{2} \\
& \mathrm{R}_{\mathrm{i}}^{2}=1-\left\{\sum_{\mathrm{k}=1}^{\mathrm{N}}\left[v_{\mathrm{i}}(\mathrm{k})-\hat{v}_{\mathrm{i}}(\mathrm{k})\right]^{2} / \sum_{\mathrm{k}=1}^{\mathrm{N}}\left[v_{\mathrm{i}}(\mathrm{k})-\bar{v}_{\mathrm{i}}\right]^{2}\right\}
\end{aligned}
$$

onde: $\hat{v}_{\mathrm{i}}(\mathrm{k})$ e $\bar{v}_{\mathrm{i}}(\mathrm{k})$ - saída estimada do elo i e média da saída real do elo i;

$\mathrm{i}=1,2-$ elos 1 e 2 do robô;

$\varepsilon_{\mathrm{i}}(\mathrm{k}+1)=v_{\mathrm{i}}(\mathrm{k})-\hat{\mathrm{v}}_{\mathrm{i}}(\mathrm{k})$ - erro de previsão do elo $\mathrm{i}$;

$\mathrm{SEQ}_{\mathrm{i}}$ - somatório do erro quadrático do elo i do robô;

$\mathrm{R}_{\mathrm{i}}^{2}$ - coeficiente de correlação múltipla do elo i do robô.

Quando o valor de $\mathrm{R}^{2}$ é igual a unidade, indica uma exata adequação do modelo para os dados medidos do processo e para $\mathrm{R}^{2}$ entre 0,9 e 1,0; o modelo pode ser considerado suficiente para muitas aplicações práticas. Valor mais baixo do SEQ para o conjunto de dados de teste indica o melhor modelo.

Os modelos matemáticos não lineares dos elos do robô manipulador em estudo são obtidos através da identificação paramétrica. Os dados que compõem o vetor de medidas, são as excitações enviadas do computador para os motores $\mathrm{CC} ; \mathrm{u}_{1}(k), \mathrm{u}_{2}(k)$, e as respostas obtidas, que são as posições angulares dos elos 1 e $2 ; v_{1}(k)=\beta_{1}(k), v_{2}(k)=\beta_{2}(k)$, conforme Eq. (5). Com a solução da Eq. (7), obtêm-se os parâmetros estimados $\hat{\theta}_{i}(k)$ dos elos 1 e 2 do robô manipulador, dados na Eq. (4).

Na geração de modelos, considerou-se: $\mathrm{m}_{1}=1, \mathrm{~d}_{1}=1, \mathrm{~m}_{2}=1, \mathrm{~d}_{2}=1, \mathrm{p}_{1}=\mathrm{p}_{2}=3$, e $\mathrm{h}_{1}=\mathrm{h}_{2}$ $=0$ e $1 ; \mathrm{m}_{1}=1, \mathrm{~d}_{1}=1, \mathrm{~m}_{2}=1, \mathrm{~d}_{2}=1, \mathrm{p}_{1}=\mathrm{p}_{2}=2$, e $\mathrm{h}_{1}=\mathrm{h}_{2}=0,1$ e 2 .

Com a adequação da Eq. (3), os parâmetros dos elos 1 e 2, são obtidos através da solução da Eq. (7) e as saídas estimadas $\hat{v}_{1}(\mathrm{k})=\hat{\beta}_{1}(\mathrm{k})$ e $\hat{v}_{2}(\mathrm{k})=\hat{\beta}_{2}(\mathrm{k})$ são obtidas pela Eq. (12).

$$
\left[\begin{array}{ll}
\hat{v}_{1}(k)=\hat{\beta}_{1}(k) \quad \hat{v}_{2}(k)=\hat{\beta}_{2}(k)
\end{array}\right]^{t}=\left[\begin{array}{ll}
\hat{\theta}_{1}(k) & \hat{\theta}_{2}(k)
\end{array}\right]^{t}[\varphi(k)]
$$


onde: $\hat{\beta}_{i}(k)$ - posiç@to angular estimada do elo i do robô;

\section{Resultados Obtidos para os Elos do Robô}

Os resultados apresentados nas Figuras 2, 3, 4 e 5, são parte dos testes realizados. A Figura 2 mostra os sinais de entrada e saída do elo 1 do robô manipulador, a Fig. 3 os sinais de entrada e saída do elo 2 . A Figura 4 mostra as saídas reais e as saídas estimadas dos elos 1 e 2 do robô manipulador e a Fig. 5 mostra as saídas reais e as estimadas dos elos do robô manipulador, no intervalo entre as amostras 200 e 230 . As saídas estimadas da Fig. 4, foram obtidas considerando o modelo não linear de grau de não linearidade de saída três para os elos 1 e 2 e horizonte igual a unidade, para os elos 1 e 2.

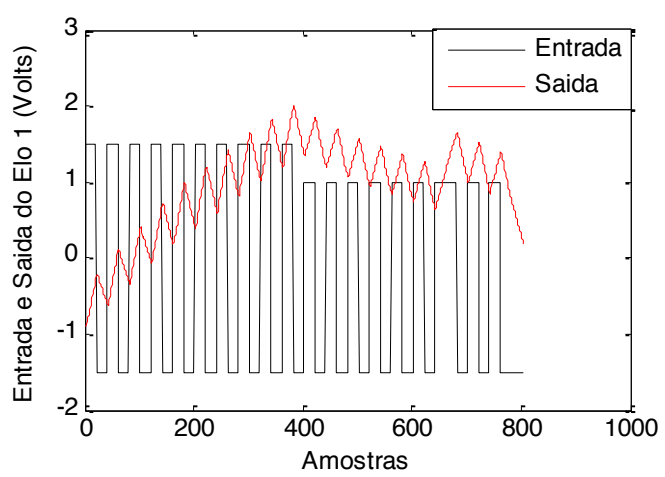

Figura 2: Entrada e saída do elo 1.

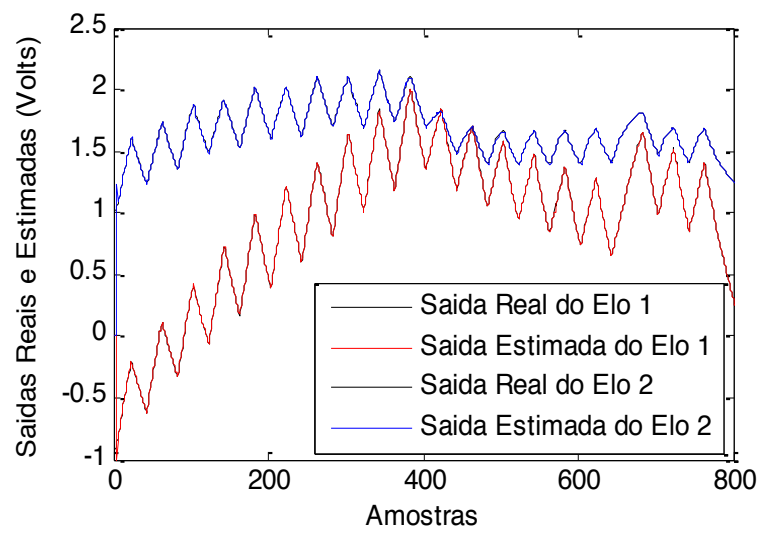

Figura 4: Saídas reais e estimadas dos elos 1 e 2 .

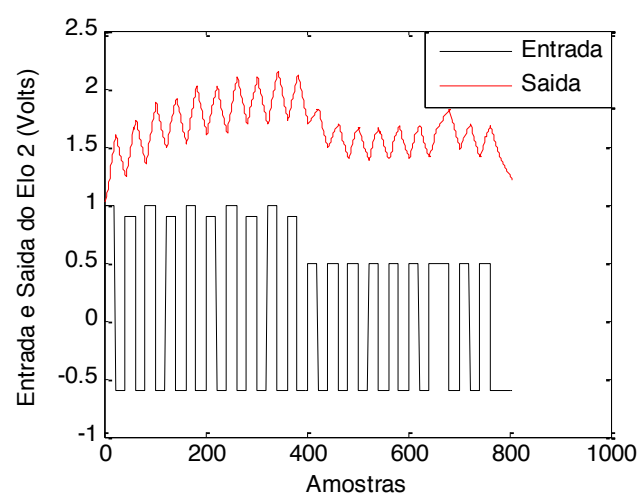

Figura 3: Entrada e saída do elo 2.

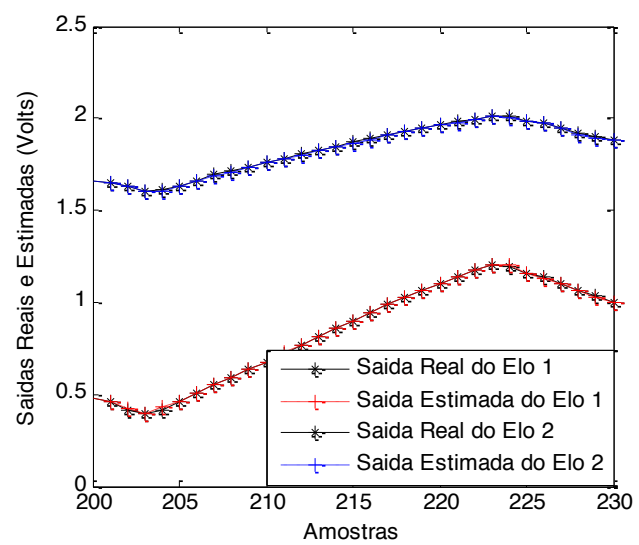

Figura 5: Saídas reais e estimadas dos elos 1 e 2 (intervalo entre 200 e 230 amostras).

Os índices de desempenho obtidos foram: $\mathrm{SEQ}_{1}=2,41, \mathrm{R}_{1}^{2}=0,99$ e $\mathrm{SEQ}_{2}=3,53$ e $\mathrm{R}_{2}^{2}=$ 
0,89. Esse modelo foi o que apresentou os melhores índices de desempenho para os elos.

\section{Conclusão}

Este trabalho apresentou técnica de identificação não linear de dois elos de um robô manipulador de cinco graus de liberdade. A identificação dos modelos foi realizada utilizando-se o algoritmo LMS, considerando a dinâmica dos dois elos do robô acoplada. Vários modelos não lineares foram testados e dos resultados obtidos, o modelo não linear de grau de não linearidade de saída três e de horizonte um, para os dois elos do robô, apresentaram os melhores índices de desempenho; o que o torna o mais indicado para implementação em controladores adaptativos.

\section{Referências}

[1] L. A. Aguirre, Introdução à Identificação de Sistemas: Técnicas lineares e não-lineares aplicadas a sistemas reais, Editora da UFMG, Belo Horizonte, MG, (2000).

[2] L. A. Aguirre, W. C. A. Amaral, M. F. M. Campos e A. P. A. Silva, Enciclopédia de Automática, Editora Blucher, São Paulo, (2007).

[3] K. J. Åstrom, B. Wittenmark, Adaptative Control, Addison Wesley, New York, (1995).

[4] A. A. R. Coelho, L. S. Coelho, Identificação de Sistemas Dinâmicos Lineares, Editora da UFSC, Florianópolis, (2004).

[5] J. J. Craig, Introduction to Robotics: Mechanics and Control, Addison Wesley, (1989).

[6] R. Isermann, Practical Aspects of Process Identification, Automática, vol. 16, 575-587, (1980).

[7] R. Isermann, K. H. Lachmann andD. Matko, Adaptative Control Systems, Prentice Hall, (1992).

[8] A. J. Koivo, T. Guo, Adaptive Linear Controller for Robotic Manipulator, IEEE Transactions on Automatic Control, vol. AC-28, 162-171, (1983).

[9] F. R. Rúbio e M. J. L. Sánchez, Control Adaptativo y Robusto, Secretariado de Publicaciones de la universidad de Sevilla, Espanha, (1996).

[10] M. W. Spong, M. Vidyasagar, Robot Dynamics and Control, John Willey \& Sons, (1989).

[11] M. C. Shih, S. I. Tseng, Identification and Position Control of a Servo Pneumático Cylinder, Control Engineering Practice, vol. 3, 1285-1290, (1995). 